\title{
Frequency Domain Based Approach for Denoising of Underwater Acoustic Signal Using EMD
}

\author{
Vijayabaskar Veeraiyan, Rajendran Velayutham and \\ Mathews M. Philip
}

\begin{abstract}
Underwater communication is usually affected by ambient noise, which may be generated by different sources, such as the wind origin sea-surface sources, ships and under water life. The properties of background noise, which are non-stationary in nature, depend on location, sea depth, wind speed and sound propagation conditions in the area. Overall performance of underwater acoustic instruments can be improved by denoising the underwater signals. This paper proposes a novel denoising method using empirical mode decomposition (EMD) technique. Frequency domain based thresholding has been used to denoise the signal, which involves three steps: (i) EMD is applied to the noisy signal to decompose the signal into intrinsic mode functions (IMFs). (ii) Thresholding is applied to each IMF in the frequency domain to remove the noise. (iii) Thresholded IMFs are added to obtain the denoised signal. Real-time experiments were performed to validate the proposed technique on the basis of the records of the ambient noise data recorded at sea for various wind speed. It was observed that the experimental results are in good agreement with the proposed algorithm under different wind-noise levels.
\end{abstract}

Keywords. Empirical Mode Decomposition, Denoising, Ambient Noise, Intrinsic Mode Functions, Threshold.

2010 Mathematics Subject Classification. 94A12.

\section{Introduction}

\subsection{Ambient Noise}

Ambient noise is a background noise in the ocean, which is due to either natural or man-made sources. Contributions from different ambient noise sources are highly variable, depending on location, deep or shallow sea, distance from the ship lane, biological diurnal and seasonal activity. In this work, wind driven ambient noise is considered, which is one of the main sources of the ambient noise for a wide frequency range [11]. The notable characteristic of the ambient sea noise field in shallow water is its high variability. It is due do this fact that at given point the noise is averaged over smaller sea surface area than in the deep ocean. Also it is 
critical, when the point of observation is at close distance to ship lanes or busy harbors, where many rapid noise sources changes their positions.

\subsection{Shallow Water}

When the ocean depth is less than 200 meter, it is considered to be shallow water. Waters close to shore and in busy harbors are dynamic locations, where rapid noise changes take place. Shallow water is notably characterized by variability, as a result of a highly variable background of ship and biological activity, high wind speeds and the level at high frequencies. When wind noise is prevalent, it is remarkably constant from site to site at the same wind speed $[8,9]$. In shallow water the propagation path is not suitable for distant shipping noise. So wind noise dominates the entire frequency range.

\subsection{Wind Noise}

In the absence of sound from ships and marine life, underwater ambient noise levels are dependent mainly on wind speeds at frequencies between $100 \mathrm{~Hz}$ and $25 \mathrm{KHz}$. Different ways by which the wind causes the ambient noise in the sea are wind turbulence, surface motion, wave interactions, spray and cavitation. All these processes are dominant in different portions of the overall frequency band from 1 to $50 \mathrm{KHz}$. Above this frequency thermal noise begins to dominate [9]. If the noise level is indeed related to wind speed, in the range of kilohertz frequencies, then it is possible to use a hydrophone as an anemometer for wind speed measurements at remote underwater locations [8]. Ocean sound in the band of $500 \mathrm{~Hz}$ to $20 \mathrm{KHz}$ is called wind noise or Knudsen noise spectra, and it was correlated very well with wind speed [6]. It was observed that there is a decrease in wind dependency of ambient noise below $500 \mathrm{~Hz}$ [11]. Dietz, Kahn, and Birch reported that in all frequency bands, at low wind speeds the average noise level was independent of wind speed, but at high wind speeds the noise level was linearly correlated with the logarithm of the wind speed.

\subsection{Denoising}

Denoising becomes a more significant process of underwater acoustics as it leads to the performance enhancement of underwater acoustic instruments. Underwater ambient noise consists of many noise signals generated by both natural and manmade sources [11]. It is important to remove ambient noise from the received acoustic signal. 
Denoising is the process of extracting the original signal from the noisy signal. Extracting the desired signal, which is buried into noise, is an essential step in the underwater communication. There are many denoising techniques available, where most of them are linear and only suitable for stationary signals. Since ambient noise is non-stationary in nature, it is important to find a suitable algorithm for denoising the non-stationary signals.

A reasonable amount of work has been done on denoising of non-stationary signals and most of them are wavelet centric. The main drawback of wavelet analysis is that it is non-adaptive in nature. Once the basic wavelet is selected, it has to be used to analyze all the data. Since the most commonly used Morlet wavelet is Fourier based, it also suffers the many shortcomings of Fourier spectral analysis. So the EMD method was considered to be the best remedy for analyzing non-linear and non-stationary data [4]. Using EMD any complicated data can be decomposed into finite number of IMFs. This decomposition method is an adaptive and therefore highly efficient. Similar to wavelet, we used EMD methods only to decompose the signal then applied different thresholding (time and frequency domain) techniques to denoise the signal. We haven't used EMD as a filtering method.

\section{The Empirical Mode Decomposition}

The data from non-linear and non-stationary processes can be analyzed using various techniques such as spectrograms, wavelet analysis and Wigner-Ville distribution, but EMD is a unique and different technique from the existing ones. EMD has been recently pioneered [4] for adaptively decomposing signals into a sum of well-behaved AM-FM components consisting of natural intrinsic building blocks that describes the complicated waveform. It does not require a priori functional basis, but the basis functions are derived adaptively from the data by the EMD sifting procedures $[4,5]$. EMD identifies the intrinsic oscillatory modes by their characteristic time scales in the data empirically, and then it decomposes the data into the corresponding IMFs through sifting process. IMF can have a variable amplitude and frequency as a function of time. It is an algorithm to assign an instantaneous frequency to each IMF, in order to decompose an arbitrary data set. The EMD method is adaptive, in which the basis of the decomposition is derived from the data, so it is different compared to all previous methods of data analysis. In the EMD approach, the data $x(t)$ is decomposed in terms of IMFs, $c_{j}$, i.e.,

$$
x(t)=\sum_{j=1}^{n} c_{j}+r_{n},
$$


where $r_{n}$ is the residue of data $x(t)$ after $n$ number of IMFs extracted. IMFs are simple oscillatory functions with varying amplitude and frequency. Hence they have the following properties [4,5]:

1. Throughout the whole length of a single IMF, the number of extrema and the number of zero-crossings must either be equal or differ at most by one (although these numbers could be differ significantly for the original data set).

2. At any data location, the mean value of the envelope defined by the local maxima and the envelope defined by the local minima is zero.

The EMD algorithm is given as follows [4,5]:

1. Given an input signal $x(t)$, identify its local extrema.

2. Interpolate between local maxima to construct an upper envelope emax $(n)$ and between local minima to construct a lower envelope emin $(n)$.

3. Compute the mean of the envelopes $m(n)=(\operatorname{emax}(n)+\operatorname{emin}(n)) / 2$.

4. Obtain $h_{1}=x(t)-m_{1}$ and inspect whether the number of extrema and the number of zero crossings are either equal or differ at most by one.

5. If not, repeat the sifting process and obtain $h_{1}-m_{11}=h_{11}$ and repeat to obtain $h_{1(k-1)}-m_{1 k}=h_{1 k}$

6. If $h_{1 k}$ constitutes an IMF, then designate it as $c_{1}=h_{1(k)}$.

7. Now we obtain the first residual $r_{1}$ via $x(t)-c_{1}=r_{1}$.

8. Treat $r_{1}$ as a new data set, and perform the sifting process to obtain $c_{2}$.

9. Continuing the sifting process we obtain $r_{2}=r_{1}-c_{2}, \ldots, r_{n-1}-c_{n}=r_{n}$.

10. The signal can be obtained from the IMF's as $x(t)=\sum_{i=1}^{n} c_{i}+r_{n}$.

\section{Existing Denoising Algorithm}

An earlier denoising algorithm was used to remove noise in ECG signal using EMD based on time domain thresholding [12]. In this work initially the same algorithm was used for denoising acoustic signal and it is considered as existing algorithm. The comparisons were done with the proposed algorithm performance. In the existing algorithm a sinusoidal signal is considered as the input test signal as shown in Figure 1 and then a real time wind driven ambient noise signal is shown in Figure 2, which has been collected at the wind speed of $5.06 \mathrm{~m} / \mathrm{s}$ and was added with the input signal to obtain the noisy signal, which is evident from Figure 3. Next to this EMD was applied to the noisy signal. The noisy signal 


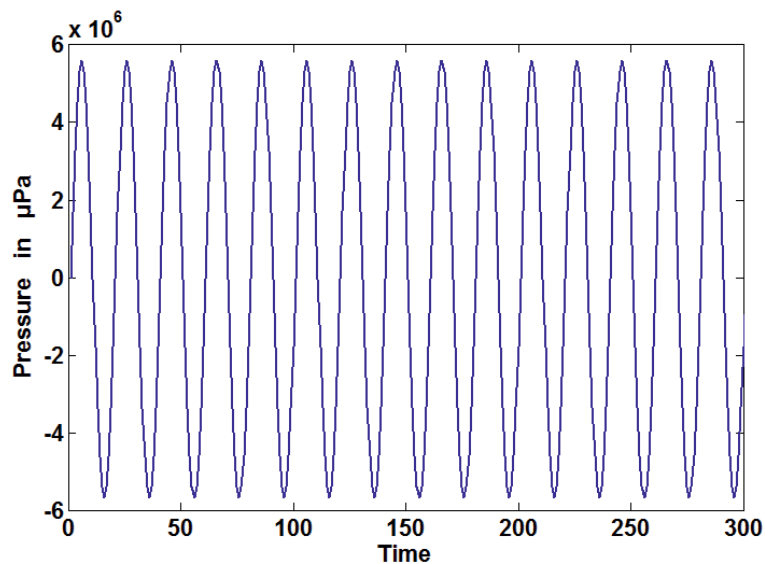

Figure 1. Input signal.

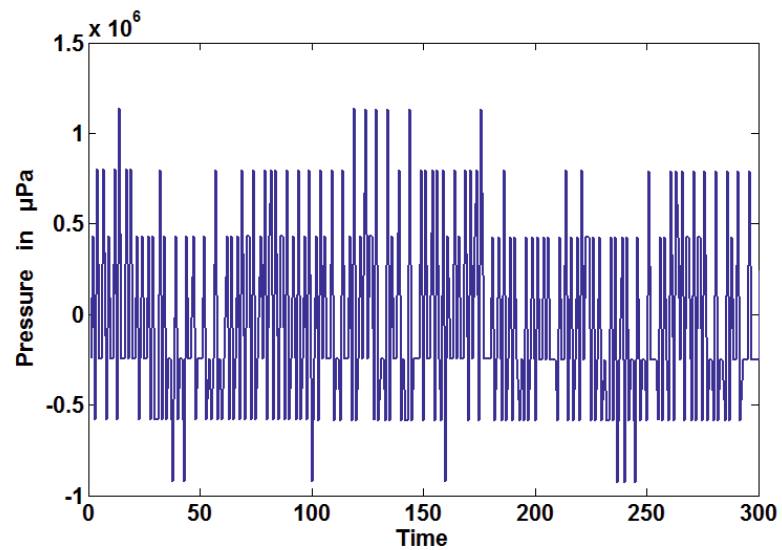

Figure 2. Noise signal.

was decomposed into a set of IMF's. The energy of each IMF was calculated as follows:

$$
\text { energy }(\text { count })=\frac{\operatorname{energy}(1)}{0.719} \cdot 2.01^{- \text {count }},
$$

where energy(1) is the energy of the first IMF which was calculated by $[1,4,5]$

$$
\operatorname{energy}(1)=\left(\frac{\operatorname{median}(|\operatorname{mode}(1)|)}{0.6745}\right)^{2},
$$

where mode(1) is the first IMF. 


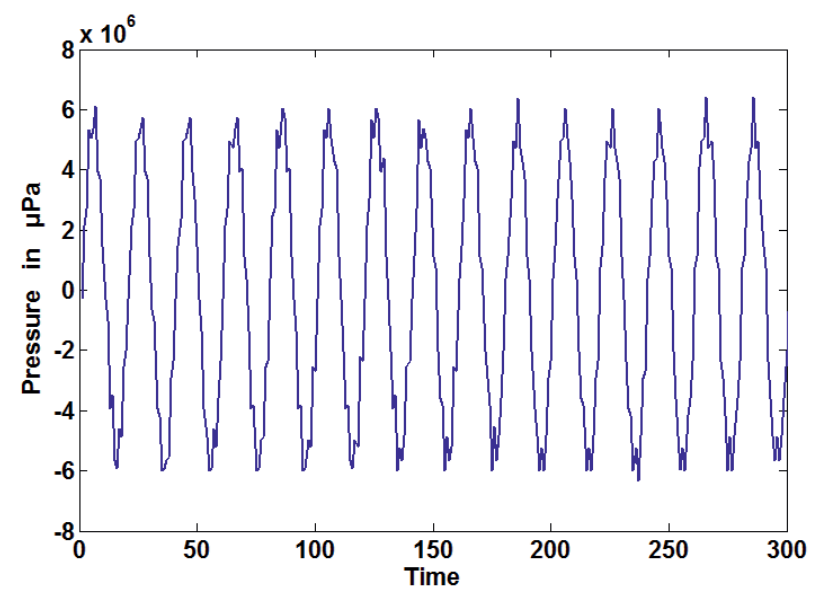

Figure 3. Noisy signal.

The threshold value of each IMF can be calculated by $[4,5,12]$

$$
\text { threshold }(\text { count })=\sqrt{\frac{\text { energy }(\text { count })}{x s i z e} \cdot 2 \cdot \log (\text { xsize })}
$$

The first IMF was discarded as it captures most of the noise and for other IMFs the adaptive threshold value was calculated using equation $(*)$.

Then the coefficients of each IMF were shrunken using a non-negative garrote threshold function which is given by $[2,3,7]$

$$
\operatorname{mode}(\text { count })=\operatorname{mode}(\text { count })-\frac{\text { threshold }(\text { count })^{\text {count }}}{\operatorname{mode}(\operatorname{count})^{(\text {count }-1)}}
$$

for values of IMF greater than or equal to the threshold value, and

$$
\operatorname{mode}(\text { count })=0
$$

for values of IMF less than the threshold value.

Finally, the shrunken IMFs were added to obtain the denoised signal, which is shown in Figure 4.

This algorithm worked well till the noise amplitude is less than the signal amplitude. When the noise amplitude is greater than half of the signal amplitude, the output signal was not satisfactory. It starts degrading as the noise amplitude increases. 


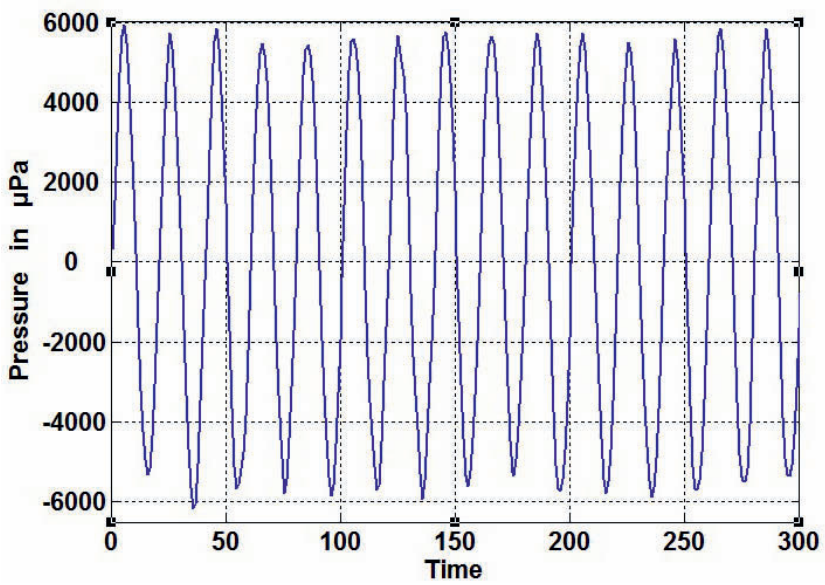

Figure 4. Denoised signal.

\section{Proposed Algorithm}

There are many methods proposed for denoising in different fields. Wavelet has been used as a tool in most of the non-linear denoising methods. In this work EMD is used as a denoising tool. Earlier denoising methods have been done using time domain thresholding. In this paper, the proposed algorithm is based on frequency domain thresholding.

The algorithm is as follows:

1. Input signal is assumed as a sinusoidal signal.

2. A real time wind noise signal at a particular wind speed was added with the input signal, which is called noisy signal.

3. EMD was applied to the noisy signal to obtain a set of IMFs.

4. Fourier transform was applied to each IMF.

5. Then the coefficients of each IMF were shrunken using the threshold function.

6. Inverse Fourier transform was applied to each IMF.

7. Then all the IMFs are added together to get the denoised signal.

The above proposed algorithm is simple and produces better results than the existing algorithm. Since the value of threshold depends on the noise signal, it performed well for different wind noise signal. 


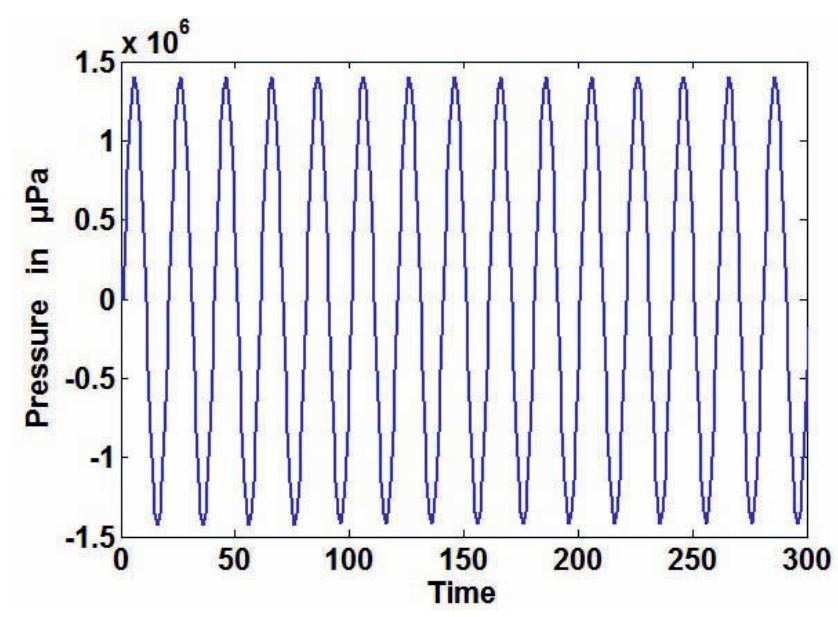

Figure 5. Input signal $(0.0053=5 \mathrm{mv})$.

\section{Results and Discussion}

Noise measurements were made using two calibrated omni directional hydrophones with a receiving sensitivity of $-170 \mathrm{~dB}$ over a frequency range between $0.1 \mathrm{~Hz}$ and $120 \mathrm{kHz}$. Data was acquired at a rate of $500 \mathrm{KHz}$ and $200 \mathrm{kHz}$, filtered and digitized with portable data acquisition system with 12-bit resolution. It was ensured that other noises were absent during the measurement. The output of hydrophone is a voltage signal, which converts the acoustic pressure into voltage, based on the sensitivity of the hydrophone. The input signal also converted into sound pressure, that is measured in micropascal, which depends on the sensitivity of the hydrophone [10]. Proposed frequency domain thresholding approach worked well for different wind noises, i.e., noise collected for various wind speed. From the results it was concluded that the algorithm works well even if the signal amplitude is well below the noise amplitude.

The signal shown in Figure 5 was considered as input signal. Figure 6 shows the real time wind driven underwater ambient noise signal, which was measured at the wind speed of $5.06 \mathrm{~m} / \mathrm{s}$. The input signal is added with the noise signal. The noisy signal is shown in Figure 7, which is more noisy compared to Figure 3. The noisy signal was decomposed into a set of IMFs by using EMD function. The IMFs are shown in Figure 8. From the figure it is clear that the IMF1 contains more noise. So we have eliminated IMF1. Then, we have applied FFT to other IMFs, which is shown in Figure 9. From the figure it is clear that IMF2 to IMF4 have more noise component and IMF5 to IMF8 have more signal component. 


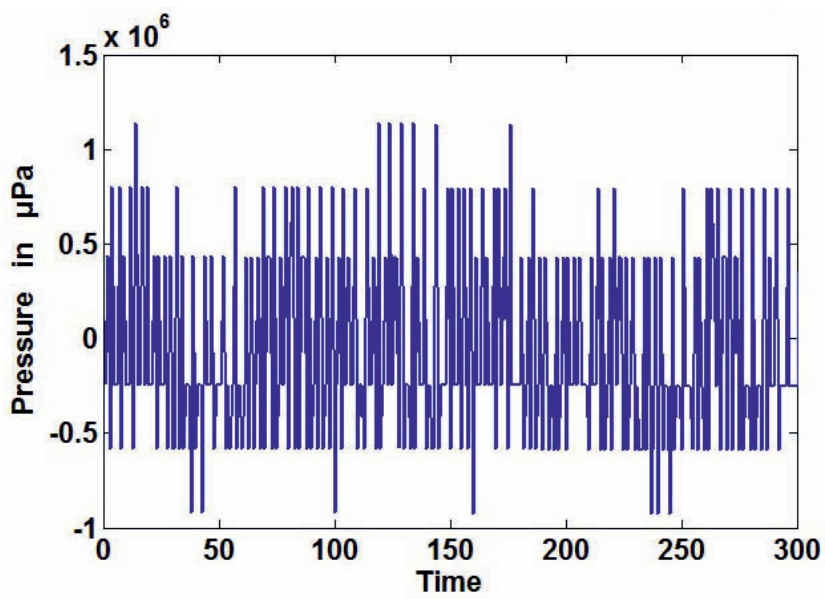

Figure 6. Noise signal.

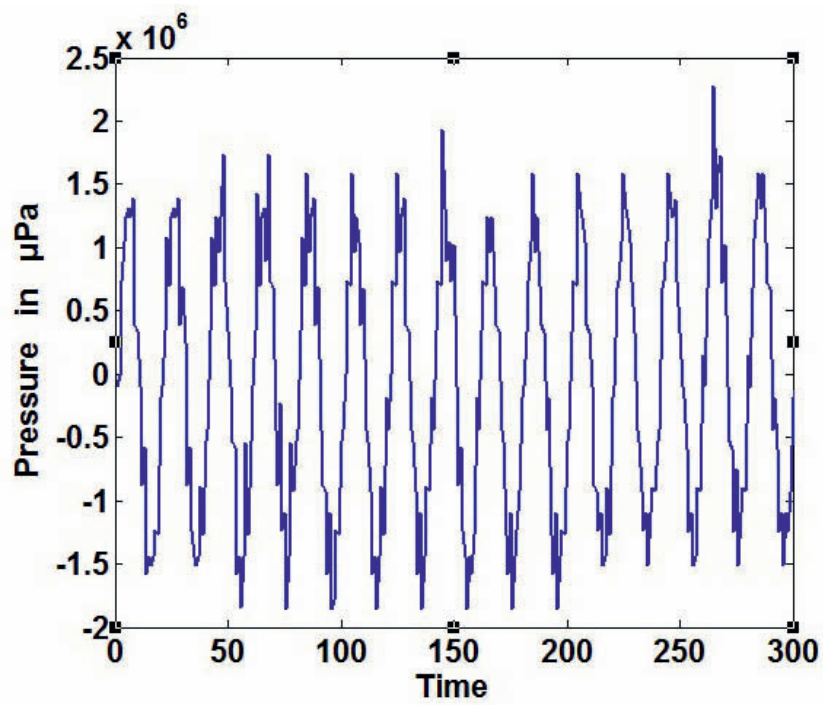

Figure 7. Noisy signal.

Different threshold values have been used and got better output for $70 \%$ to 90\% thresholding, i.e., in each IMF, the signals, which have FFT amplitude below threshold value, were assigned zero. After applying threshold, IFFT was taken to each IMF, and then all the thresholded IMFs were added to get the denoised signal. The denoised signal was shown in Figure 10 for different threshold values. 

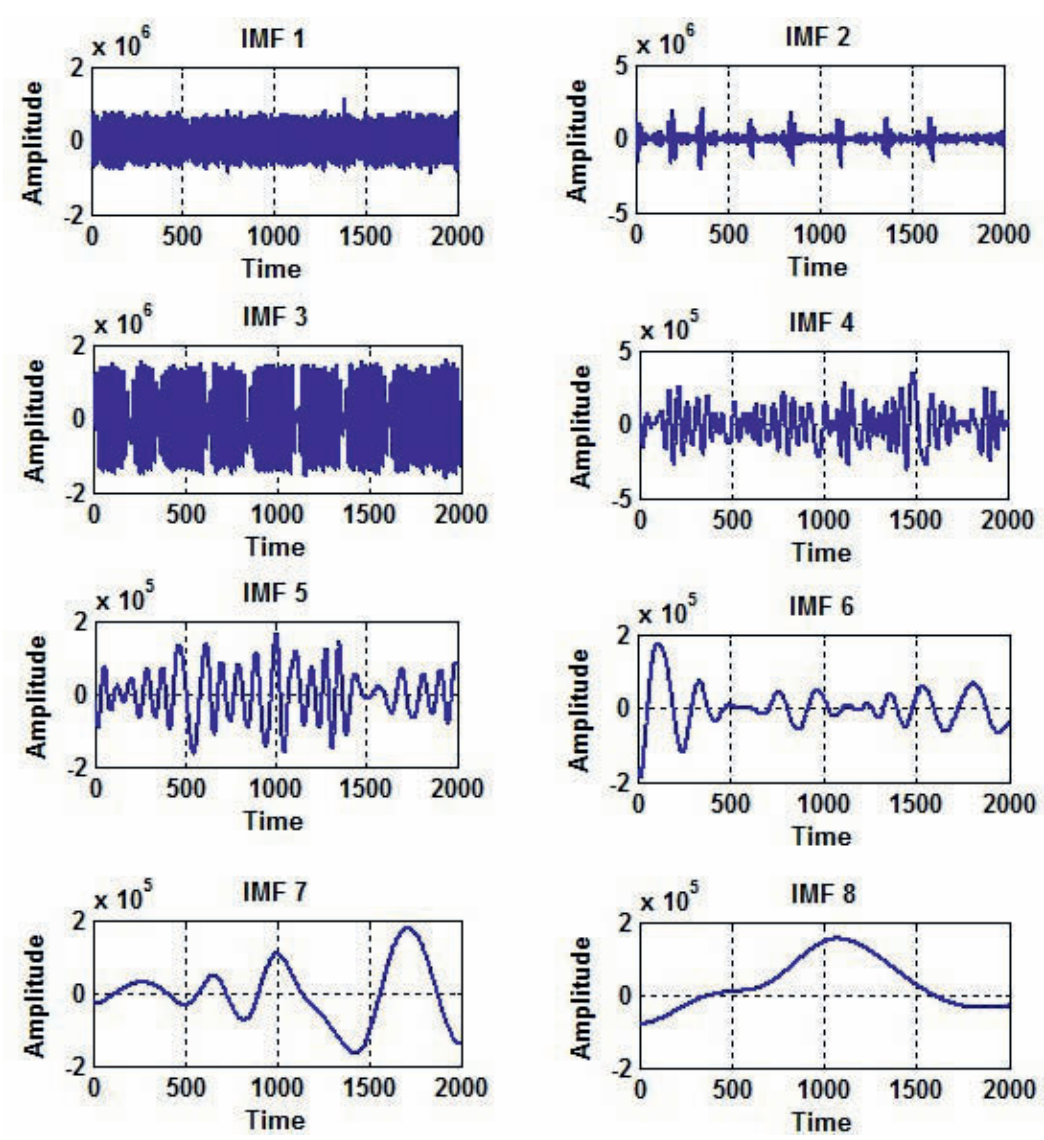

Figure 8. IMFs of noisy signal.

The out was good for the threshold values of $70 \%$ and above. At $90 \%$ threshold the output resembles the input signal well. Compared to the existing time domain thresholding algorithm, the proposed frequency domain thresholding algorithm gives the better results. In the existing algorithm, the signal amplitude was considered as $20 \mathrm{mv}$, which is shown in Figure 1. In the proposed algorithm, the signal amplitude was considered as $5 \mathrm{mv}$, which is shown in Figure 5. In the existing algorithm, the denoised signal amplitude, which is shown in Figure 4, was much lesser than the actual input signal and also the output not exactly resembles the input signal, which is shown in Figure 1. But in the proposed algorithm the amplitude of denoised signal (at 90\% threshold), shown in Figure 10, is same as the input signal, which is shown in Figure 5 and also the denoised signals resembles the input signal well. In all the figures, the signal amplitude is represented 

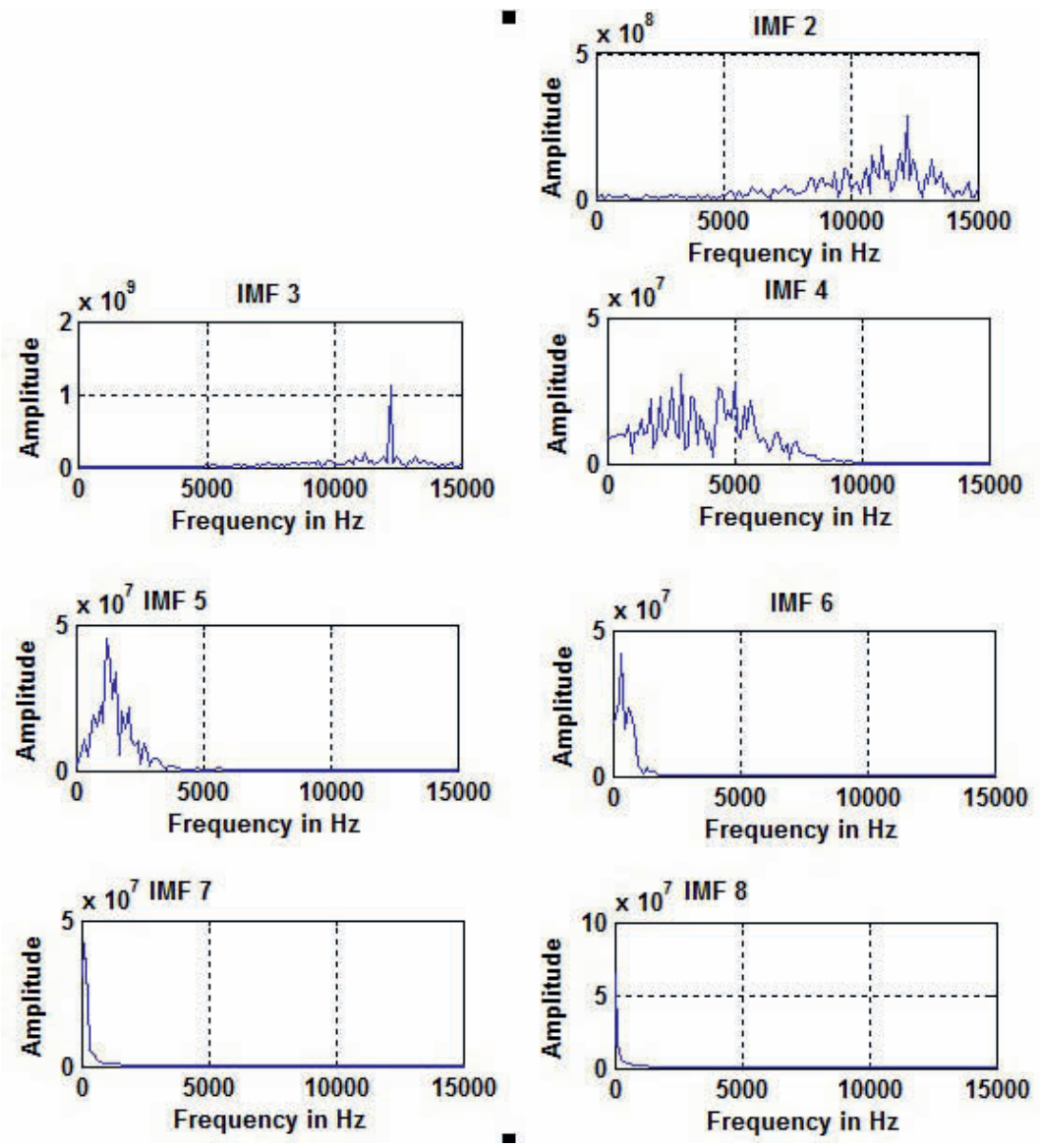

Figure 9. FFT of IMFs.

in micropascal, i.e., volt is converted into micropascal based on sensitivity of the microphone. This result again reveals the reliability of the algorithm for different wind noise signals. In order to validate the algorithm the mean square error (MSE) was calculated using the following equation:

$$
\text { Mean Square Error (MSE) }=\frac{1}{N} \sum_{n=1}^{N}(Z(n)-\hat{Z}(n))^{2},
$$

where $N$ is the length of data, $Z(n)$ is the actual input signal and $\hat{Z}(n)$ is the denoised signal. Table 1 gives the MSE value for different threshold values. From the table it is clear that the MSE value decreases as the threshold value increases 

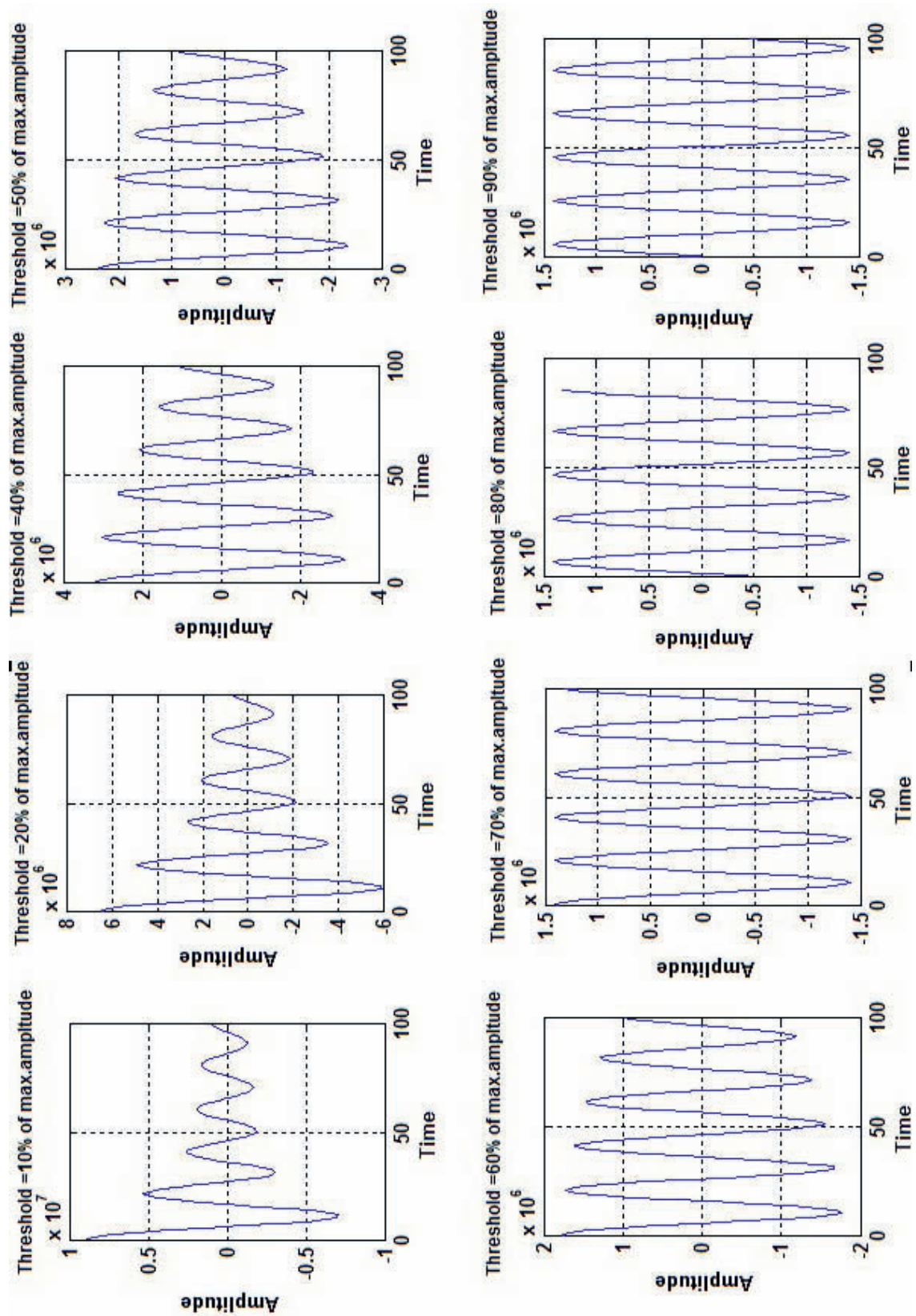

Figure 10. Denoised signal. 


\begin{tabular}{cc}
\hline Threshold value in $\%$ & MSE value \\
\hline 20 & 0.0056 \\
40 & 0.0032 \\
50 & 0.0029 \\
60 & 0.0019 \\
70 & 0.0011 \\
80 & 0.0011 \\
90 & 0.0011 \\
\hline
\end{tabular}

Table 1. MSE value for different threshold.

\begin{tabular}{lll}
\hline $\begin{array}{l}\text { Wind speed } \\
\text { in } \mathrm{m} / \mathrm{s}\end{array}$ & $\begin{array}{l}\text { MSE value in } \\
\text { time domain }\end{array}$ & $\begin{array}{l}\text { MSE value in } \\
\text { frequency domain }\end{array}$ \\
\hline 5.06 & 0.0035 & 0.0011 \\
\hline
\end{tabular}

Table 2. Comparison of MSE values in time and frequency domain.

and there is no change in the MSE value for above $70 \%$ of threshold. Table 2 shows the MSE value for two different denoising methods. The MSE value in frequency domain is less compared to the time domain approach. The MSE value was calculated for different wind speeds and it was good in the frequency domain approach. It is concluded that the proposed frequency domain thresholding algorithm produces better results compared to the existing algorithm. This algorithm has been developed and tested for wind noise and therefore can be extended to include other constituents of ambient noise.

Acknowledgments. The authors wish to acknowledge Dr. Ram Bilas Pachori, Assistant Professor, Electrical Engineering, Indian Institute of Technology Indore, for his valuable support and guidance.

\section{Bibliography}

[1] A. O. Boudraa, J. C. Cexus and Z. Saidi, EMD-based signal noise reduction, Int. J. Signal Process. 1 (2004), 33-37.

[2] D. L. Donoho, De-noising by soft thresholding, IEEE Trans. Inf. Theory 41 (1995), 613-627. 
[3] H.-Y. Gao, Wavelet shrinkage denoising using the non-negative garrotte, J. Comput. Graph. Stat. 7 (1998), 469-488.

[4] G. Huang et al., The empirical mode decomposition and the Hilbert spectrum for nonlinear and non-stationary time series analysis, Proc. R. Soc. London, Ser. A 454 (1998), 903-995.

[5] N. E. Huang and S. S. Shen, Hilbert-Huang Transform and Its Applications, Interdisciplinary Mathematical Sciences 5, World Scientific Publishing, Singapore, 2005.

[6] V. O. Knudsen, R. S. Alford and J. W. Emling, Underwater ambient noise, J. Mar. Res. 7 (1948), 410-429.

[7] Y. Kopsinis, Development of EMD-based denoising methods inspired by wavelet thresholding, IEEE Trans. Signal Process. 57 (2009), 1351-1362.

[8] C. L. Piggott, Ambient sea noise at low frequencies in shallow water of the Scotian shelf, J. Acoustic Soc. Am. 36 (1964), 2152-63.

[9] R. J. Urick, Ambient Noise in the Sea, Peninsula Publishing, Washington, 1984.

[10] V. Vijayabaskar and V. Rajendran, Analysis and modeling of wind dependence of ambient noise in shallow water of arabian sea, Europ. J. Sci. Res. 50 (2011), 28-34.

[11] G. M. Wenz, Acoustic ambient noise in the ocean: Spectra and sources, J. Acoustic Soc. Am. 34 (1962), 1936-1956.

[12] Z. Zhidong, L. Yi and L. Qing, Adaptive noise removal of ECG signal based on ensemble empirical mode decomposition, in: Adaptive Filtering Applications (2011), $123-140$.

Received November 17, 2012.

\section{Author information}

Vijayabaskar Veeraiyan, Sathyabama University, Chennai, India.

E-mail: v_vijaybaskar@yahoo.co.in

Rajendran Velayutham, SSN College of Engineering, Chennai, India.

E-mail: drvrajen@gmail.com

Mathews M. Philip, Sathyabama University, Chennai, India.

E-mail: mathewsmphilip3@gmail.com 